\title{
Spontaneous Resolution of a Traumatic Cataract in Cerebral Palsy Libyan Patient a Case Report
}

\author{
Khalid A k Elmajri* \\ Department of Ophthalmology, Omer almuktar University, Libya
}

*Corresponding author: khalid A K elmajri, Department of Ophthalmology, Omer amuktar university, Derna, Libya.

Received Date: August 02, 2021

Published Date: September 13, 2021

\begin{abstract}
The rate of surgery in eastern Mediterranean countries remains low. The provision of widespread cataract surgery delivered in a timely fashion before individuals are visually impaired by this condition may result for a reduction of blindness and visual loss. A related disturbing issue is the frequency with which ophthalmic surgery results in intra operative or postoperative complications. In several eastern Mediterranean countries, the rate of complications following cataract surgery remains high. The recent introduction of new techniques for cataract surgery, including phacoemulsification, has been started by ophthalmologists in eastern Mediterranean countries. Despite these advances, less than $20 \%$ of the cataract surgery in eastern Mediterranean countries is phacoemulsification. This is because of lack of resources in certain areas for cold and emergency cases, the presence of corneal scars, and advanced mature cataracts [1].
\end{abstract}

\section{History}

A 10-year-old male came as emergency case to ophthalmology clinic in a teaching Alwahda Derna hospital with trauma right eye by spoon food accidentally by his sister for evaluation of a possible open globe and management. The boy complained of pain, was taken to the local emergency treatment center for evaluation. A slit lamp examination failed to reveal a foreign body. Early the next morning he awoke with worsening pain. On examination by ophthalmologist noted a small corneal laceration 2 to $3 \mathrm{~mm}$ at 8 to 9 - 0 clock and lenticular change (cataract).

Past Ocular History: No prior ocular injury, surgery, or illness.

Past Medical History: known case of cerebral palsy

Medications: *tobradex ophthalmic drops, $4 \mathrm{x} /$ day, in the right eye.

*Tobradex eyey ointment, twice dialy

*Pilocarpin eye drops, three time daily (although the incision was small but taken this decision to prevent iris prolapse through the incision )
*Vegamox eye drops, five time dialy

Family and Social History: Noncontributory

Exam, Ocular:

$\neg \quad$ Motility: Normal; no restrictions

$\neg \quad$ Slit lamp examination

- $\quad \mathrm{mm}$ diameter full- thickness stellate corneal laceration (Seidel negative)

- $\quad$ Anterior chamber - deep

- Anterior capsular no tear

- $\quad$ Posterior rosette-shaped subcapsular cataract

$\neg \quad$ Dilated fundus exam (DFE): was difficult because cataracts changes of the lens

$\neg \quad$ Ultrasound. Noforeign body. No vitreous hemorrhage ,the posterior lens capsule appeared to be intact (Figure $1 \& 2$ ). 


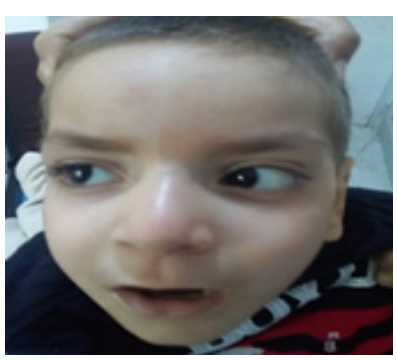

Figure 1:

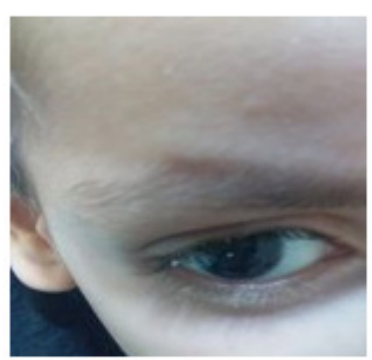

Figure 2:

Follow up after three days, a rosette-shaped PCC was observed while the anterior capsule and lens nucleus were still same picture and the small corneal lacerated wound healed and pilocarpin stopped. The lens of the left eye was normal. Within another 3 days, the PCC increased in density and covered the whole pupillary area. Fundoscopic examination of the right eye were difficult to perform secondary to the cataract, however, no gross abnormalities were noted. Options for management of the cataract, including cataract surgery, were discussed with the patient's father. Considering his age and his general condition we agreed upon conservative observation. The patient returned for follow-up every 3 week and waited for an appropriate time for surgery. At the two-month followup, we observed that the size of the PCC began to decrease. Five months later, only a faint imprint of the original cataract remained.

Based on the patient's age and special his satiation and difficulty to surgical intervention, the patient received conservative management and follow-up. Interestingly, Surprisingly, the cataract gradually spontaneously resolved with time over the following 5 months and the fundus appear clear and restored red reflex (Figure 3):

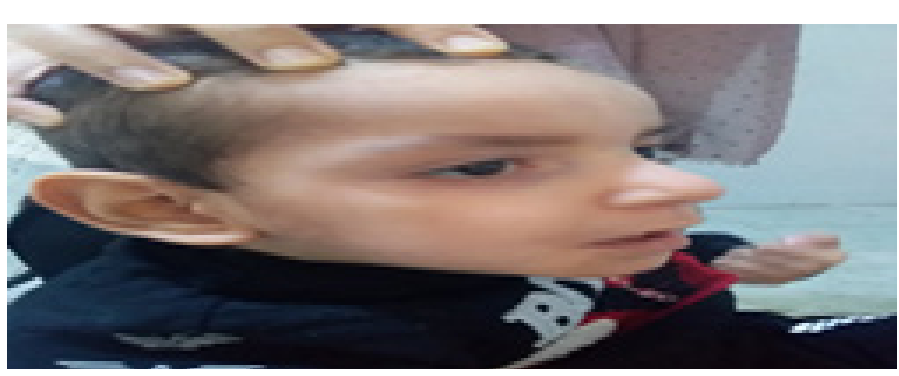

Figure 3:

\section{Discussion}

Although in traumatic cataract, cataract extraction, usually in combination with intraocular lens implantation, is required for vision correction [2,3]. Spontaneous resolution of posterior cortical cataract (PCC) has been reported previously in cases where the cataract is caused by uncontrolled acute hyperglycemia [4] In the case of traumatic cataracts, spontaneous reversal of PCC mainly occurs with mild or limited ocular injury, such as mild blunt contusion or small intralenticular foreign body $[5,6]$. or transient feathering of the lens after intraocular gas tamponade [7]. In severe open-globe ocular injury cases, spontaneous resolution of extensive dense PCC is rare [8].

However, if the lenticular capsule damage is limited, the proliferative lens epithelium can reseal the wound without progressive cataract formation, and in rare cases, allow cataract resolution [5]. The lens epithelium and fiber cells become damaged during injury, disrupting the integrity and permeability of the lenticular capsule. This leads to an influx of aqueous humor into the 
lens lamella, causing it to swell and irreversibly opacify secondary to the denaturation of lens proteins [9]. Rapidly developing traumatic cataract is a common manifestation in ocular injury cases, such as intra lenticular foreign body, contusive injury and open-globe ocular injury. Brini A. et al. inferred that the resorption of small superficial lens opacities may occur in a pseudo-lysosomal fashion [10]. Neumayer T. et al. observed the changes and disappearance of lens vacuoles within 4 weeks in PCC patients, which might partially explain PCC spontaneous regression [11].

Paradoxically, in our case, cataract density was worst at the posterior cortex and not the anterior part of lens. Previous reports indicate that in blunt ocular trauma, posterior capsular rupture is more likely to appear in children and young adults [12,13] Wolter proposed that the absence of a sclerotic nucleus and strong zonular fibers in children and teenagers may allow the force of impact to transmit to the posterior part of lens [14]. In open-globe injury cases, cataract formation results from direct impact, and the location of the most dense opacity commonly indicates the point of greatest impact $[3,11]$. We hypothesize that in this open-globe injury case, the PCC is secondary to indirect shock waves stemming from impact.

In young cataract patients, treatment options are more debated and require further consideration. It is known that the critical period of eye development ranges from 2 to 6 months of age, and emmetropization is generally achieved by 9 years of age [15]. To avoid amblyopia, some ophthalmologists recommend early surgical intervention for pediatric ocular traumatic cataracts, and in simple PCC cases without corneal or retinal injury, cataract extraction with intraocular lens implantation can restore excellent visual acuity [2, 16]. However, in young patients, cataract extraction also results in the loss of accommodation. For children, loss of accommodation and physiologic hyperopic reserve might affect the progress of emmetropization [17]. For teenagers, this loss of accommodation aggravates asthenopia during near work in daily life.

Trauma is a cause of monocular blindness in the developed world, although few studies have addressed the problem of trauma in rural areas. The aetiology of ocular injury in rural areas is likely to differ from that in urban areas and is worthy of investigation [18]. Any strategy for prevention requires knowledge of the cause of injury, which may enable more appropriate targeting of resources towards preventing such injuries. Eye trauma represents a large, potentially preventable burden on both victims and society as a whole [19]. Importantly, conservative observation may greatly benefit the young patient by avoiding unnecessary invasive surgical intervention, and its complication and subsequent loss of vision.

\section{Conclusion}

several critical factors taken in consideration when treatment traumatic cataract in pediatric such as:

$\neg \quad$ 1-general condition of patient $\neg \quad 2$ - age

$\neg$ 3- The availability and extent of the possibility of performing the operation

$\neg$ 4- visual development and the preservation of accommodative potential.

$\neg \quad 5$ - type and extend of trauma

To optimize treatment in pediatric traumatic cataract, conservative management with lens preservation is important to consider in young, traumatic cataract patients where invasive surgical intervention may not be required.

\section{Acknowledgement}

None.

\section{Conflict of Interest}

No conflict of interest.

\section{References}

1. Ayed S, Daghfous F, Guermazi K, et al. (1991) Causes of blindness in Tunisian children. Rev Int Trach Pathol Ocul Trop Subtrop Sante Publique 68: 123-128

2. Singh RB, Thakur S, Ichhpujani P (2018) Traumatic rosette cataract. BM] Case Rep 11(1): e227465.

3. Du Y, He W, Sun X, Lu Y, Zhu X (2018) Traumatic cataract in children in eastern China: Shanghai pediatric cataract study. Sci Rep 8(1):2588.

4. Ramkumar H, Basti S (2008) Reversal of bilateral rosette cataracts with glycemic control. Scientific World Journal 8: 1150-1151.

5. Rofagha S, Day S, Winn BJ, Ou JI, Bhisitkul RB, et al. (2008) Spontaneous resolution of a traumatic cataract caused by an intralenticular foreign body. J Cataract Refract Surg 34(6): 1033-1035

6. Avasthy P, Gupta RB (1958) Traumatic cataract. Br J Ophthalmol 42(4): 240-241.

7. Petermeier K, Szurman P, Bartz-Schmidt UK, Gekeler F (2010) Pathophysiology of cataract formation after vitrectomy. Klin Monatsbl Augenheilkd 227(3): 175-180.

8. Moreschi C, Da Broi U, Lanzetta P (2013) Medico-legal implications of traumatic cataract. J Forensic Legal Med 20(2): 69-73.

9. Shah MA, Shah SM, Shah SB, Patel CG, Patel UA (2011) Morphology of traumatic cataract: does it play a role in final visual outcome? BMJ Open 1(1): e000060.

10. Brini A, Porte A, Stoeckel ME (1963) Resorption of necrotic lens material by a newly formed lens capsule in certain types of cataract. Nature 200: 796-797.

11. Neumayer T, Hirnschall N, Georgopoulos M, Findl O (2017) Natural course of posterior subcapsular cataract over a short time period. Curr Eye Res 42(12): 1604-1607.

12. Bansal A, Fenerty CH (2020) Spontaneous resolution of a rapidly Formed dense cataract following Nd: YAG laser peripheral Iridotomy in a case of Pigmentary Glaucoma. J Glaucoma 29(4): 322-325.

13. Matalia J, Kasturi N, Anaspure H, Shetty BK, Matalia H (2015) Isolated posterior capsular split limited by Weiger's ligament after blunt ocular trauma in a child mimicking posterior lenticonus. J AAPOS 19(6): 557558.

14. Lee SI, Song HC (2001) A case of isolated posterior capsule rupture and traumatic cataract caused by blunt ocular trauma. Korean J Ophthalmol 15(2): 140-144 
15. Wolter JR (1963) Coup-contrecoup mechanism of ocularinjuries. Am Ophthalmol 56: 785-796.

16. khokhar SK, Pillay G, Dhull C, Agarwal E, Mahabir M, Aggarwal P (2017) Pediatric cataract. Indian J Ophthalmol 65(12): 1340-1349.

17. Medina A (2018) Prevention of myopia by partial correction of hyperopia: a twins study. Int Ophthalmol 38(2): 577-583.
18. Khatry SK, Lewis AE, Schein OD, M D Thapa, E K Pradhan, et al. (2004) The epidemiology of ocular trauma in rural Nepal. Br J Ophthalmol 88: 456-460.

19. Alfaro DV, Jablon EP, Rodriguez Fontal M, Simon J Villalba, Robert E Morris, Michael Grossman, et al. (2005) Fishing-related ocular trauma. Am J Ophthalmol 139: 488-492. 